$01,07,11,19$

\title{
Откольная прочность ударно-разогретого циркония и фазовая диаграмма в области существования его полиморфных модификаций высокого давления
}

\author{
(С) А.М. Молодец ${ }^{1}$, А.А. Голышев ${ }^{1}$, Д.В. Шахрай ${ }^{1}$, Д.Ю. Ковалёв ${ }^{2}$ \\ ${ }^{1}$ Институт проблем химической фризики РАН, \\ Черноголовка, Россия \\ ${ }^{2}$ Институт структурной макрокинетики и проблем материаловедения им. А.Г. Мержанова Российской академии наук, \\ Черноголовка, Россия \\ E-mail: molodets@icp.ac.ru
}

Поступила в Редакцию 26 июля 2019 г.

В окончательной редакции 26 июля 2019 г.

Принята к публикации 15 августа 2019 г.

\begin{abstract}
Определена откольная прочность циркония, разогретого сильной ударной волной до тысячных температур и испытавшего превращения в более плотные полиморфные модификации. Представлены измеренные профили давления на границе образец-мягкая преграда в откольных экспериментах при плоском одномерном нагружении. Построены уравнения состояния трех полиморфных модификаций циркония в диапазоне давлений до $50 \mathrm{GPa}$. Рассчитаны термодинамические состояния циркония в условиях выполненных экспериментов, что в комплексе с результатами измерений профилей давления позволило определить откольную прочность циркония, разогретого в цикле ударное сжатие-разгрузка. Величина откольной прочности циркония составила $-2.8(7) \mathrm{GPa}$ при температуре $1027(70) \mathrm{K}$. Представлен анализ экспериментальных профилей давления с привлечением результатов математического моделирования проведенных экспериментов.
\end{abstract}

Ключевые слова: откол, цирконий, полиморфизм, ударные волны, фазовая диаграмма.

DOI: 10.21883/FTT.2020.01.48734.564

\section{1. Введение}

Ядерная энергетика предъявляет особые требования к теплофизическим свойствам материалов в широком диапазоне силовых и температурных нагрузок. Спектр материалов ядерной энергетики охватывает практически все классы твердых тел, включая чистые металлы и сплавы, стали, расплавы, керамики, стекла и др. Среди металлических материалов важное место занимают переходные металлы IV группы Ti, Zr, Hf. Эти металлы является функциональными и конструкционными материалами атомной промышленности и в ряде случаев должны работать в условиях высоких динамических нагрузок и гарантировать минимальные последствия возможных аварийных событий, приводящих к экстремальным ударно-волновым воздействиям на детали ядерных установок. Поэтому прикладные исследования теплофизических свойств металлических материалов ядерных энергетических установок в условиях высоких температур и давлений сопровождаются фундаментальными исследованиями их физико-механических свойств и превращений при высоких статических и динамических давлениях.

Высокоскоростное разрушение in situ во встречных волнах разгрузки (откольное разрушение) циркония изучалось в $[1,2]$ с использованием оптических методик регистрации скорости свободной поверхности образца. В этих работах изучалась зависимость откольной проч- ности циркония от амплитуды нагружающей ударной волны в диапазоне 4-10 GPa. Кроме этого, в [1] исследовалось влияние температуры образца на величину откольной прочности предварительно нагретых образцов до $640 \mathrm{~K}$, а также полиморфного превращения циркония в области $10 \mathrm{GPa}$. При этом в [1] отмечается, что исследование откола in situ с помощью оптических методик регистрации скорости свободной поверхности при более высоких температурах, характерных для температур эксплуатации деталей из циркониевого сплава, ограничивается потерей отражательной способности циркония. Температура на уровне $600 \mathrm{~K}$ оказывается предельной и для емкостной методики [3] при исследовании температурной зависимости in situ откольной прочности из-за электрического пробоя и усложнения экспериментальной сборки огнеупорными материалами. Однако эти ограничения могут быть преодолены, если использовать ударный разогрев образца вместе с данными по регистрации давления на границе образца с мягкой средой. Действительно, в методическом отношении методики, опирающиеся на измерение скорости поверхности и давления на границе с мягкой средой одинаковы (см. [4] и ссылки в ней). Дополнительная трудность во втором случае обусловлена лишь необходимостью построения фазовой траектории ударного сжатия и разгрузки в области существования полиморфных фаз высокого давления циркония. В данной работе представлены результаты исследования откольной прочности 
циркония с использованием специально разработанных уравнений состояния фаз высокого давления циркония и соответствующих фазовых траекторий аналогично тому, как это сделано для гафния в [5].

\section{2. Образцы, схема ударно-волнового нагружения и результаты эксперимента}

Образцы для ударно-волновых экспериментов изготавливались из технического циркония марки 702, и представляли собой пластины толщиной $10 \mathrm{~mm}$, большие плоские поверхности которых шлифовалась до величины шероховатости $R_{z}=10 \mu \mathrm{m}$. Массовая доля примесей в образцах составляла $\mathrm{Hf}-4.5 \%, \mathrm{Fe}-0.2 \%, \mathrm{O}-0.16 \%$, $\mathrm{N}-0.025 \%, \mathrm{Cr}-0.02 \%$.

Плотность образцов составила $\rho_{0}=6.64(5) \mathrm{g} / \mathrm{cm}^{3}$. Продольная скорость звука при нулевом давлении равна $C_{l}=4.76(5) \mathrm{km} / \mathrm{s}$. Для объемной скорости звука $C_{b}$ использовалось значение $C_{b}=3.91 \mathrm{~km} / \mathrm{s}$ для циркония Э110 из [1].

Рентгенофазовый анализ проводился на дифрактометpe ДРОН-3М на излучении $\mathrm{Cu} K_{\alpha}$ с монохроматором на вторичном пучке. Регистрация рентгенограмм велась в режиме пошагового сканирования в интервале углов $2 \theta=30-90^{\circ}$, шаг $0.02^{\circ}$, время набора $2 \mathrm{~s}$. Рентгенограмма 1 исходного образца, представленная на рис. $1, a$, свидетельствует, что основным компонентом материала является гексагональная фаза $\alpha-\mathrm{Zr}$ поскольку угловое положение дифракционных линий соответствует данным для этой фазы из базы ICDD (PDF2 card № 05-0665). Соотношение интенсивностей рефлексов [002] и [101] свидетельствует о наличии текстуры в образце, то есть о преимущественной ориентировке плоскостей 002 зерен относительно поверхности шлифа.

Текстура образцов в состоянии поставки присутствует не только на поверхности, но и на поверхностях разрезов исходных образцов, т.е. образцы в исходном состоянии были текстурированы по всему объему.

Металлографические исследования проводили на оптческом микроскопе. Травление шлифов велось в 10\% водном растворе плавиковой кислоты. Металлографический шлиф исходного образца показан на рис. 2, a. Как видно, исследуемые образцы первоначально имели примерно равноосную микроструктуру с размером зерна $20-40 \mu \mathrm{m}$.

В ударно-волновых экспериментах по исследованию откольной прочности использовалась традиционная методика образец-,мягкая“ преграда (см., например, [4], а также [5]). Здесь ударник 1 , разогнанный продуктами взрыва до скорости $W_{0}$, соударялся с исследуемым образцом 2 и генерировал в нем плоскую ударную волну. Образец соприкасался с мягкой преградой 3. На границе раздела 2-3, ударная волна распадалась на встречную волну разгрузки, уходящую в образец, и
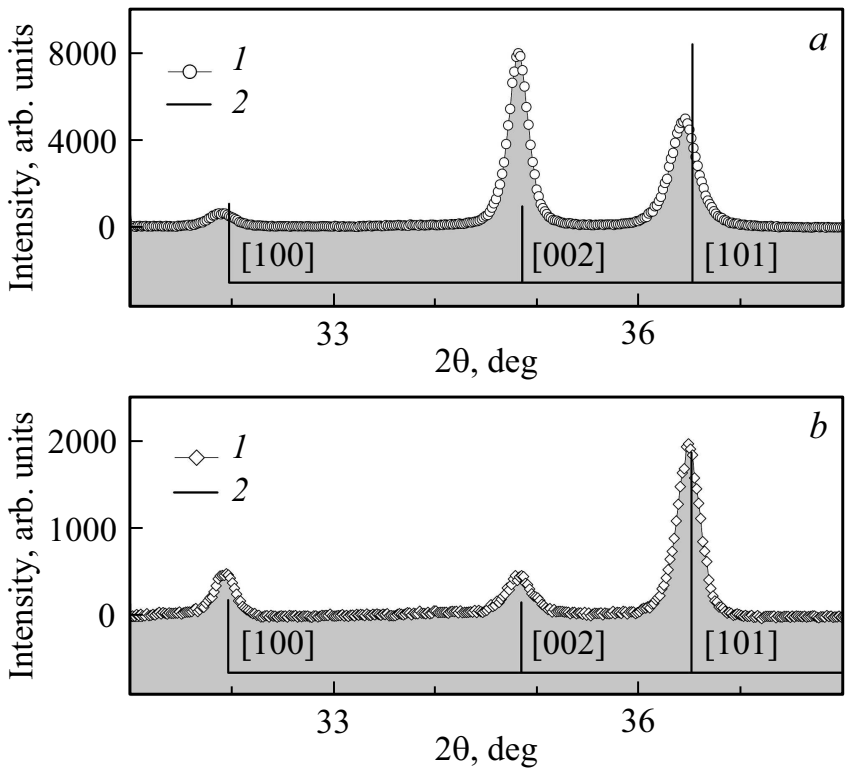

Рис. 1. Рентгенограммы поверхностей образцов циркония марки 702. $a-$ рентгенограмма внутреннего слоя исходного образца в состоянии поставки (1), рентгенограмма № 05-0665 из базы данных ICDD $\alpha$-фазы циркония (в квадратных скобках указаны индексы плоскостей $\alpha$-фазы) (2); $b$ - рентгенограмма поверхности откола образца циркония, полученная по схеме pис. $3, a(1), 2-$ то же, что и на рис. $1, a$.

волну сжатия, распространяющуюся в преграду. В эксперименте регистрировалось падение электрического напряжения $U$ на чувствительном элементе манганинового датчика 4 во время прохождения по нему волны сжатия. Электрическое напряжение пересчитывалось в электросопротивление датчика, а затем в профиль $P(t)$ (зависимость давления $P$, от времени $t$ ) с помощью калибровки манганинового датчика из [6].

Откольное разрушение образца возникало в области взаимодействия встречной волны разгрузки с проходящей волной разгрузки от тыльной стороны ударника. Информация об откольном разрушении образца отражалась на профиле $P(t)$ в форме откольного импульса, что позволяло определить откольную прочность образца.

На рис. 3, $a$ „мягкой“ преградой служил плексиглас (РММА). На рис. $3, b$,мягкой“ преградой являлась слоистая преграда, состоявшая из изолирующей прослойки 3, в виде двух тефлоновых пленок, склеенных вакуумной смазкой (ТВС [6] среда) и алюминиевой пластины 5. Как и в [5], использование тефлона позволило исключить паразитные поляризационные сигналы, имевшие место при использовании РММА. Использование алюминия позволило получить экспериментальные данные по откольному разрушению образца циркония в усложненных условиях слоистой преграды.

На рис. 4, $a$ представлен профиль $P(t)$ в экспериментах по схеме рис. $3, a$, на рис. $4, b$ и $c$ представлены профили $P(t)$ в экспериментах по схеме рис. $3, b$. 

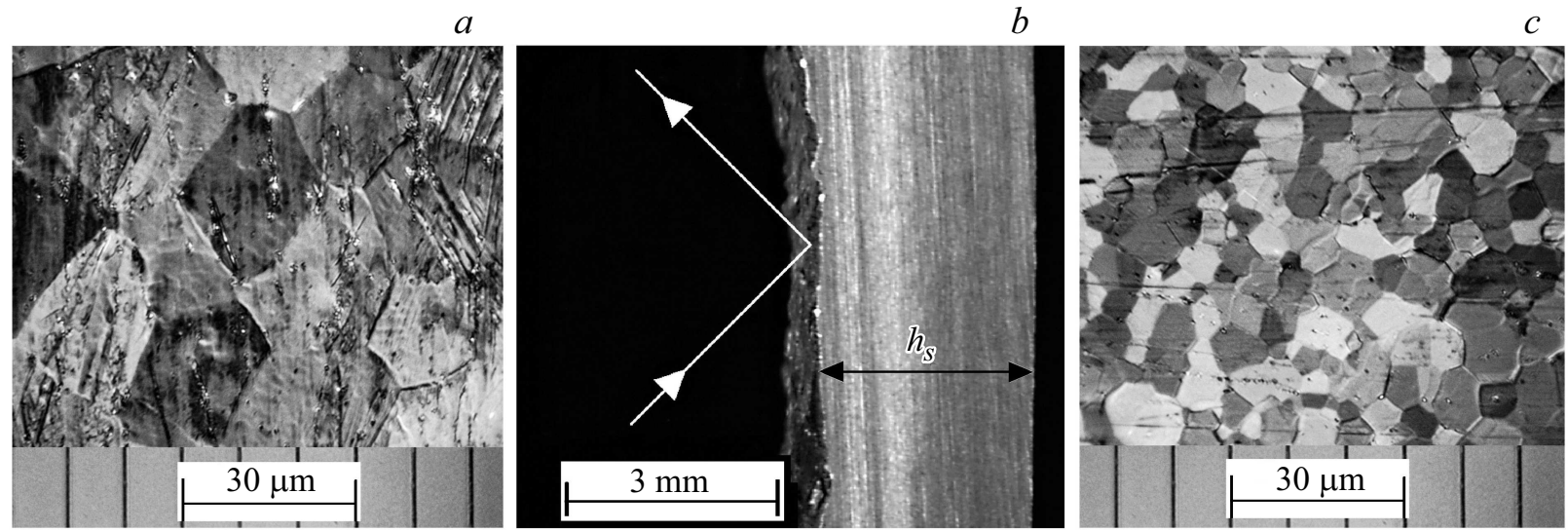

Рис. 2. Микроструктура циркония марки 702. $a$ - исходный образец; $b-$ сечение откольной пластины образца перпендикулярное плоскости удара (направление удара - слева направо), $h_{S}$ - толщина откольной пластины, наклонными стрелками указано направление рентгеновского пучка при снятии рентгенограммы с поверхности откола, противоположной поверхности контакта образца с плексигласом; $c$ - микрошлиф сечения откольной пластины.

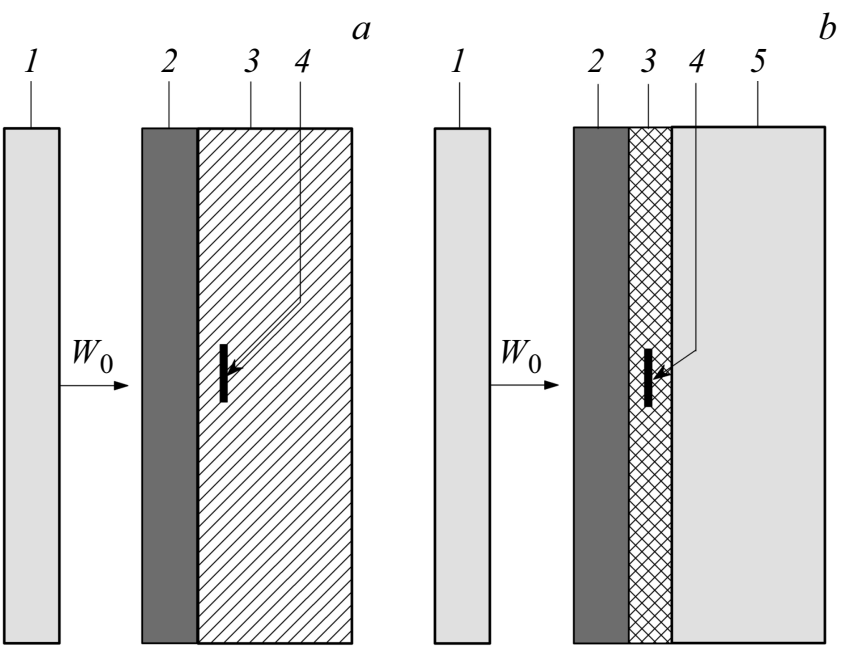

Рис. 3. Схема нагружения образцов циркония по регистрации профилей давления на границе образцов циркония с мягкой средой. $a: 1-$ стальной (нержавеющая сталь 12Х18Н10Т) ударник толщиной $h_{0}=4.0(1) \mathrm{mm}$, разогнанный до скорости $W_{0}=2.45(1) \mathrm{km} / \mathrm{s}, 2$ - образец циркония 702 толщиной $H_{0}=10.00(5) \mathrm{mm}, 3$ - пластина плексигласа (РММА) толщиной 10.0(1) mm, 4 - манганиновый датчик толщиной $0.03 \mathrm{~mm}$ располагался на расстоянии $2 \mathrm{~mm}$ поверхности образца; $b$ : 1 - то же что и на рис. $3, a, 2$ - образец циркония 702 толщиной $H_{0}=10.00(5) \mathrm{mm}$ или $H_{0}=4.00(5) \mathrm{mm}, 3-$ изолирующая прослойка из ТВС среды толщиной $0.4 \mathrm{~mm}, 4-$ манганиновый датчик толщиной $0.03 \mathrm{~mm}$, расположенный на расстоянии $0.2 \mathrm{~mm}$ от поверхности образца, 5 - алюминиевая пластина толщиной 14(1) $\mathrm{mm}$.

В экспериментах по схеме рис. 3, $a$ осуществлялось сохранение образца после ударного нагружения для последующего рентгенофазового и металлографического анализа. Результаты исследования сохраненных образцов представлены на рис. $1, b, 2, b, c$ соответственно.

\section{3. Обсуждение экспериментальных профилей давления и определение откольной прочности}

Экспериментальный профиль 1 на рис. 4, $a$ содержит псевдопредвестник перед фронтом ударной волны. Этот предвестник обусловлен поляризационной наводкой от плексигласа, искажающей первичный электрический сигнал $U$ манганинового датчика. Использование в качестве материала преграды неполярного материала (тефлона) приводит, как это видно на рис. 4, $b$, к исчезновению этого псевдопредвестника.

Вместе с этим на экспериментальном профиле 1 рис. $4, b$ следует отметить появление двухволновой конфигурации фронта ударной волны. Моделирование этого эксперимента (см. раздел 4.3), дающее модельный профиль 2, позволяет установить, что эта конфигурация фронта обусловлена реверберацией ударной волны в ТВС среде между циркониевым образцом и алюминиевой пластиной.

Моделирование позволяет также установить, что в результате этой реверберации в образец входит короткая волна разрежения, которая в дальнейшем может приводить к отколу в циркониевом образце. Эта ситуация реализовывалась в экспериментах по схеме рис. $3, b$ при толщине образца $H_{0}=10.00(5) \mathrm{mm}$. На рис. 4,c представлен соответствующий экспериментальный профиль 1 и два модельных профиля 2 и 3. Профиль 2 („безоткольный“ профиль) соответствует бесконечно большой прочности циркония, а профиль 3 получен при значении откольной прочности $\sigma_{S}=-3 \mathrm{GPa}$. Сопоставление профилей $1-3$ позволяет идентифицировать малозаметную особенность на экспериментальном профиле 1 в области $t_{S}$ как откольное разрушение образца в усложненной постановке рис. $3, b$. 
Возвращаясь к рис. 4, $a$, отметим, что экспериментальный профиль $P(t)$ на этом рисунке содержит характерные значения $P_{1}$ и $P_{2}$, которые служат базовыми величинами при определении откольной прочности $\sigma_{S}$ образца. Так, величина $\sigma_{S}$ может быть определена графически (см., например, [4]), если известны $P_{1}, P_{2}$, ударные адиабаты образца и преграды, а также их изэнтропы разгрузки в координатах давление $(P)$-массовая скорост $(u)$. Для целей данной работы необходимые ударные адиабаты и изэнтропы разгрузки рассчитывались на основе разработанных уравнений состояния (см.
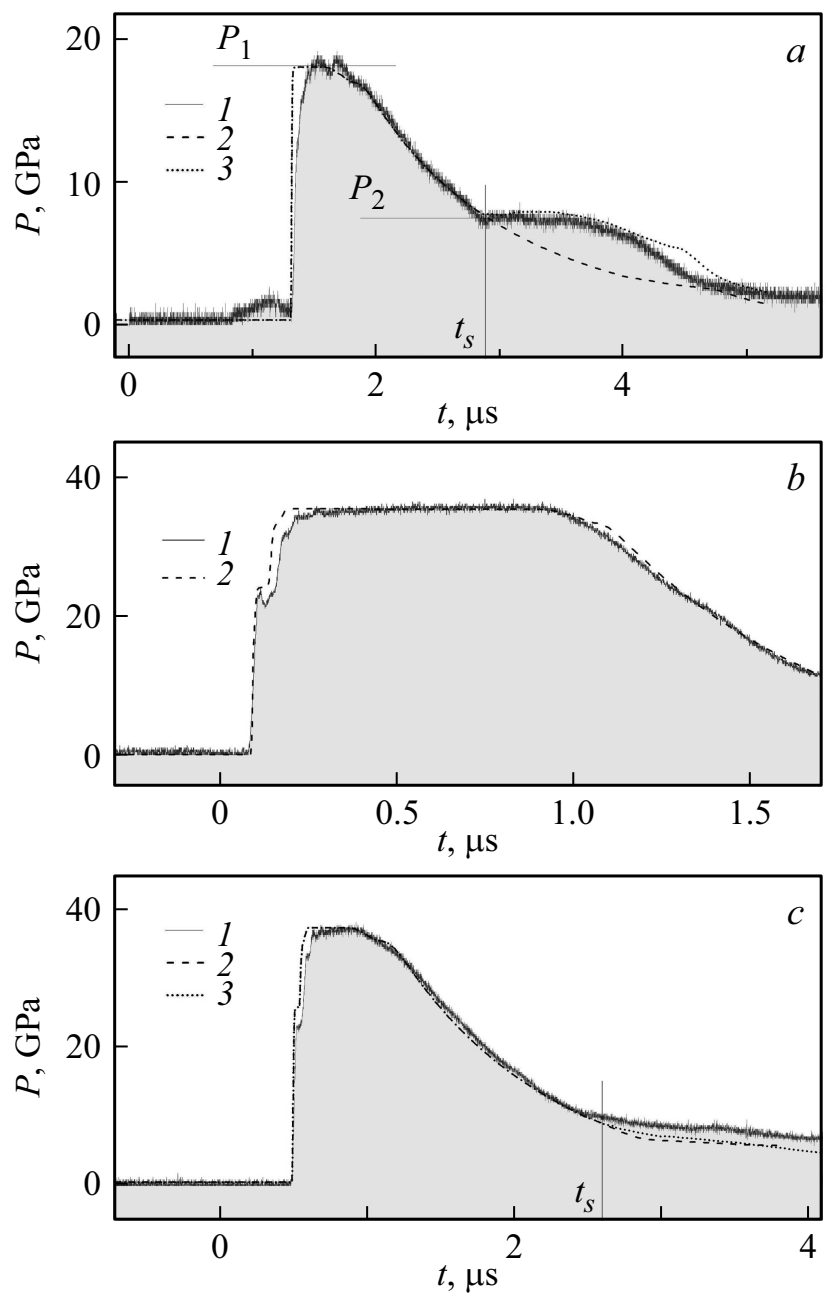

Рис. 4. Профили давления $P(t)$ в экспериментах по схеме pис. 3. $a-$ эксперимент по схеме рис. $3, a: 1-$ экспериментальный профиль для образца толщиной $H_{0}=10.00(5) \mathrm{mm}$, $P_{1}$ - максимальное значение $P, P_{2}-$ значение $P$ в момент $t_{S}$ (момент прихода откольного импульса на манганиновый датчик), 2 - „безоткольный“ модельный профиль, 3 - модельный профиль при значении откольной прочности $\sigma_{S}=-3 \mathrm{GPa}$; $b$ - эксперимент по схеме рис. $3, b$ для образца толщиной $H_{0}=4.00(5) \mathrm{mm}: 1$ - экспериментальный профиль, 2 модельный профиль; $c$ - эксперимент по схеме рис. $3, b$ для образца толщиной $H_{0}=10.00(5) \mathrm{mm}: 1-$ экспериментальный профиль, 2 - „безоткольный“ модельный профиль, 3 модельный профиль при $\sigma_{S}=-3 \mathrm{GPa}$.

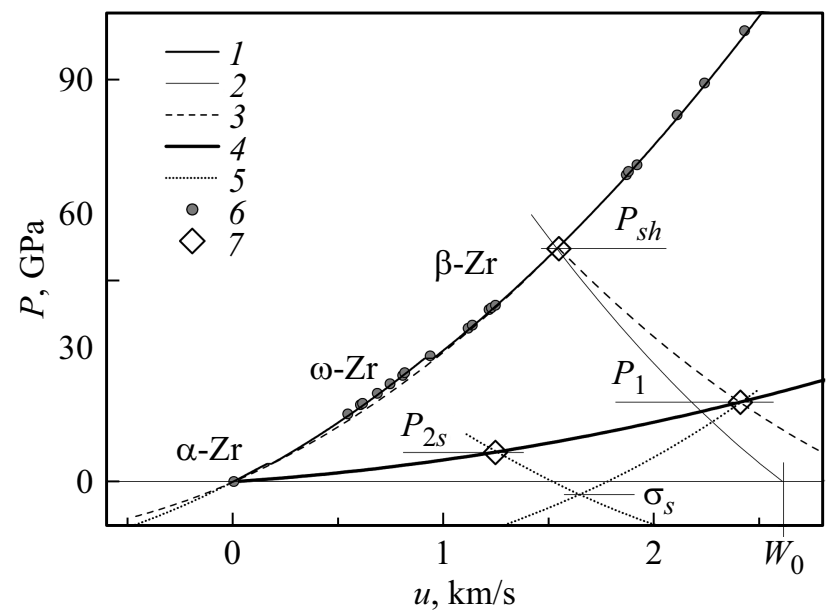

Рис. 5. Диаграмма давление $(P)$-массовая скорость $(u)$ ударного сжатия и разгрузки циркония при отколе. 1 расчетная ударная адиабата циркония, 2 - ударная адиабата торможения ударника из нержавеющей стали с начальной скоростью $W_{0}, 3$ - изэнтропы разгрузки $\beta-\mathrm{Zr}, 4-$ ударная адиабата плексигласа, совпадающая с его изэнтропой разгрузки, 5 - изэнтропы разгрузки $\alpha-\mathrm{Zr}, 6$ - экспериментальная ударная адиабата циркония из [7], $7-$ состояния циркония в экспериментах данной работы.

разд. 4.1). Результаты этих расчетов представлены на рис. 5 соответствующими графиками.

Рассмотрим с учетом этих ударных адиабат и изэнтроп, а также данных рис. 4, а графическое определение откольной прочности $\sigma_{S}$ на диаграмме рис. 5. Здесь пересечение ударных адиабат образца 1 и ударника 2 определяет состояние ударно-сжатого циркония в ударной волне с амплитудой $P_{s h}=52(1) \mathrm{GPa}$ в области $\beta$-Zr. После распада ударной волны на границе образецпреграда реализуется состояние с давлением $P_{1}$ на изэнтропе циркония $\beta-\operatorname{Zr} 3$ и ударной адиабате плексигласа 4. Давление $P_{1}$, регистрируемое манганиновым датчиком, берется из рис. $4, a$. В дальнейшем давление в плексигласе уменьшается вдоль изэнтропы плексигласа до величины $P_{2 S}$, соответствующей моменту прихода откольного импульса.

Величина давления $P_{2 S}=P_{2}-\Delta P_{2}$ на рис. 5 получается с использованием значения $P_{2}$ из рис. $4, a$ с учетом гистерезиса манганинового датчика в разгрузке, а также упруго-пластической поправки [8] в виде $\Delta P_{2}=h_{S}\left(1 / C_{b}-1 / C_{l}\right)$, где значение толщины откольной пластины $h_{S}=3.0(1) \mathrm{mm}$ определено с помощью шлифа рис. $2, b$.

Через состояния плексигласа при $P_{1}$ и $P_{2 S}$ проведены изэнтропы разгрузки $\alpha-\mathrm{Zr}$ циркония 5 , пересечение которых определяет значение откольной прочности циркония $\sigma_{S}=-2.8(7) \mathrm{GPa}$. В величину погрешности $\sigma_{S}$ включены все перечисленные неопределенности базовых величин.

Таким образом, данные рис. $2, b, 4, a$ и 5 позволяют заключить, что откольная прочность циркония, предвари- 
тельно сжатого ударной волной амплитудой 52(1) $\mathrm{GPa}$, составляет величину $\sigma_{S}=-2.8(7) \mathrm{GPa}$.

Отметим, что изэнтропы 3 и 5 разгрузки $\beta-\mathrm{Zr}$ и $\alpha-\mathrm{Zr}$ соответственно на рис. 5 незначительно отличаются друг от друга в диапазоне давлений до $-3 \mathrm{GPa}$. В соответствии с этим при определении откольной прочности в графических построениях на рис. 5 для определенности использовалась изэнтропа разгрузки фазы $\alpha-\mathrm{Zr}$.

Во введении отмечалось, что в данной работе использовались мощные ударные волны для сильного ударного разогрева образца с последующей оценкой температур образца в области растяжения на основе уравнений состояния циркония. Ниже приведены техника построения полуэмпирических уравнений состояния полиморфных фаз циркония и соответствующие расчеты.

\section{4. Уравнения состояния, фазовая диаграмма циркония и моделирование эксперимента по откольному разрушению}

\section{1. Уравнения состояния полиморфных модификаций циркония}

Как известно (см. [9] и ссылки в ней) цирконий при нормальных условиях имеет гексагональную плотноупакованную ГПУ (hcp)-структуру ( $\alpha$-Zr-фаза). При температуре $1136 \mathrm{~K}$ и атмосферном давлении происходит полиморфное превращение $\alpha-\mathrm{Zr}$ в $\beta$-Zr-фазу с объемно центрированной кубической ОЦК (bcc) упаковкой атомов. При увеличении давления вдоль комнатной изотермы $\alpha$-Zr-фаза превращается сначала в $\omega$-Zr-фазу с гранецентрированной кубической ГЦК (fcc) при давлении на уровне $3.4 \mathrm{GPa}$, а затем при давлении $32-35 \mathrm{GPa}$ $\omega$-Zr-фаза превращается в $\beta$-Zr-фазу.

В данной работе индивидуальные уравнения состояния для каждой полиморфной модификации циркония рассчитывались на основе полуэмпирического выражения для свободной энергии всесторонне сжатого изотропного твердого тела $\Phi=\Phi(V, T)$, представляющего сумму фононной $F=F(V, T)$ и электронной $F_{e}=F_{e}(V, T)$ составляющих

$$
\Phi=F(V, T)+F_{e}(V, T) .
$$

Формульный инструментарий и техника построения уравнений состояния взяты из [5]. Так, фононная составляющая, базирующаяся на модели эйнштейновских осцилляторов, имеет вид

$$
F=3 R\left[\frac{\Theta}{2}+T \ln \left(1-\exp \left(-\frac{Q}{T}\right)\right)\right]+E_{x},
$$

где $R$ - удельная газовая постоянная, $V$ - удельный объем материала, $T$ - его температура, $\Theta=\Theta(V)-$ объемная зависимость характеристической температуры, определяемая согласно как

$$
\begin{gathered}
\Theta=\Theta_{0}\left(\frac{v_{0}-V}{v_{0}-V_{0}}\right)^{2}\left(\frac{V_{0}}{V}\right)^{2 / 3}, \\
v_{0}=V_{0}\left(1+\frac{2}{\gamma_{0}-2 / 3}\right) .
\end{gathered}
$$

В (1)-(4) $\Theta_{0}=\Theta\left(V_{0}\right)$ - характеристическая температура, $\gamma_{0}=\gamma\left(V_{0}\right)$ - термодинамический коэффициент Грюнайзена, $V_{0}$ - удельный объем в начальных условиях. Под начальными условиями $\left(T_{0}, P_{0}\right)$ везде ниже подразумевается состояние с комнатной температурой $T_{0}$ и атмосферным давлением $P_{0}$.

Объемная зависимость потенциальной энергии $E_{x}=E_{x}(V)$ также взята из [5] в виде

$$
\begin{gathered}
E_{x}=-v_{x}\left(C_{1} H_{x}+C_{2} x\right)+C_{3}+E_{m}, \\
H_{x}=9\left(\frac{1}{10} x^{-\frac{2}{3}}+2 x^{\frac{1}{3}}+\frac{3}{2} x^{\frac{4}{3}}-\frac{1}{7} x^{\frac{7}{3}}+\frac{1}{70} x^{\frac{10}{3}}\right), \\
x=\frac{V}{v_{x}} .
\end{gathered}
$$

В (5)-(7) $v_{x}$ - подгоночный параметр, первое приближение которого есть $v_{0}$. Уточнение величины $v_{x}$ производится из условия наилучшего совпадения расчетной комнатной изотермы или ударной адиабаты с экспериментальными данными. Параметры $C_{1}, C_{2}, C_{3}$ константы, выражающиеся через справочные свойства материала (плотность, модуль объемного сжатия, характеристическая температура, коэффициент Грюнайзена при начальных условиях) и подгоночный параметр $v_{x}$. Постоянное слагаемое $E_{m}$ задает уровень отсчета энергии для рассматриваемых фаз высокого давления.

Электронная составляющая $F_{e}$ записывалась согласно литературным данным [10]

$$
F_{e}=-\frac{1}{2} \beta_{0} T^{2}\left(\frac{V}{V_{0}}\right)^{1 / 2}
$$

где $V_{0}$ - начальный удельный объем, $\beta_{0}$ - коэффициент электронной теплоемкости.

Уравнения состояния находились с помощью частных производных (1) согласно общим термодинамическим соотношениям. Калорическое уравнение состояния (зависимость внутренней энергии $E$ от объема и температуры) определяется частной производной свободной энергии по температуре $E=\Phi-T(\partial \Phi / \partial T)$, которое после дифференцирования приобретает вид

$$
E=E_{x}+3 R \Theta\left(\frac{1}{2}+\frac{1}{\exp (\Theta / T)-1}\right)+T^{2} \beta_{0}\left(\frac{V}{4 V_{0}}\right)^{1 / 2}
$$

Термическое уравнение состояния (зависимость давления $P$ от объема и температуры $T$ ) определяется частной производной свободной энергии по объему 
$P=P(V, T)=-\partial \Phi / \partial V$, которое после дифференцирования приобретает вид

$$
\begin{gathered}
P=C_{1} \frac{d H_{x}}{d x}+C_{2}+3 R \frac{\gamma}{V} \Theta\left(\frac{1}{2}+\frac{1}{\exp (\Theta / T)-1}\right)+P_{e} \\
\gamma=\frac{2}{3}+\frac{2 V}{v_{0}-V}, \\
P_{e}=\frac{\beta_{0}}{V_{0}}\left(\frac{T}{2}\right)^{2}\left(\frac{V_{0}}{V}\right)^{1 / 2} .
\end{gathered}
$$

Таким образом, термодинамическое описание $(1)-(12)$ содержит параметры $V_{0}, v_{0}, \Theta_{0}, \beta_{0}$ для тепловой составляющей и параметры $v_{x}, C_{1}, C_{2}, C_{3}, E_{m}$ для потенциальной составляющей свободной энергии. При этом основным подгоночным параметром служит $v_{x}$. Нахождение перечисленных параметров для полиморфных модификаций циркония представлено ниже.

Основным состоянием циркония считается $\alpha-\mathrm{Zr}$-фаза co справочными значениями $V_{0}, v_{0}, \Theta_{0}$ и величиной $E_{m}=0$. Величины $V_{0}, v_{0}$, определялись с использованием результатов [9]. Величина $\Theta_{0}$ подбиралась так, чтобы расчетное значение теплоемкости было равно справочному значению $25.61 \mathrm{~J} / \mathrm{mol} \mathrm{K}$. Коэффициент электронной теплоемкости $\beta_{0}$ в (8) для $\alpha-Z r$ считался известным и был взят из справочной литературы $\beta_{0}=2.77 \mathrm{~mJ} / \mathrm{mol} \mathrm{K}$.

Величина $v_{x}$ для фазы $\alpha-\mathrm{Zr}$ уточнялась с использованием экспериментальной комнатной изотермы этой фазы из $[9,11]$. При этом расчетная изотерма рассчитывалась с помощью уравнения состояния (10), в котором значение $T$ считалось постоянным и равным значению комнатной температуры $T_{0}$. На рис. 6, $a$ показано соответствие расчетной и комнатной изотерм высокого давления для $\alpha-\mathrm{Zr}$. Найденная величина $v_{x}$ вместе с формульной молярной массой $M_{f}$, а также $V_{0}, v_{0}, \Theta_{0}$ и $C_{1}, C_{2}, C_{3}$ для полиморфной модификации циркония $\alpha$-Zr представлены в табл. 1,2 .

Таблица 1. Параметры для тепловых составляющих свободной энергии (1) $\alpha-\mathrm{Zr}, \omega-\mathrm{Zr}$ и $\beta-\mathrm{Zr}$-фаз циркония

\begin{tabular}{c|c|c|c|c|c}
\hline Фаза & $\begin{array}{c}M_{f}, \\
\mathrm{~g} / \mathrm{mol}\end{array}$ & $\begin{array}{c}V_{0}, \\
\mathrm{~cm}^{3} / \mathrm{mol}\end{array}$ & $\begin{array}{c}v_{0}, \\
\mathrm{~cm}^{3} / \mathrm{mol}\end{array}$ & $\begin{array}{c}\Theta_{0}, \\
\mathrm{~K}\end{array}$ & $\begin{array}{c}\beta_{0}, \\
\mathrm{~mJ} / \mathrm{mol} \mathrm{K}\end{array}$ \\
\hline$\alpha-\mathrm{Zr}$ & 91.22 & 14.022 & 60.596 & 217.5 & 2.77 \\
$\omega-\mathrm{Zr}$ & 91.22 & 13.838 & 113.631 & 209.2 & 0.0 \\
$\beta-\mathrm{Zr}$ & 91.22 & 14.050 & 593.332 & 170.0 & 2.77
\end{tabular}

Таблица 2. Параметры для потенциальной энергии (7) $\alpha-Z r$, $\omega$-Zr и $\beta$-Zr-фаз

\begin{tabular}{c|c|c|c|c|c}
\hline Фаза & $\begin{array}{c}v_{x}, \\
\mathrm{~cm}^{3} / \mathrm{mol}\end{array}$ & $\begin{array}{c}C_{1}, \\
\mathrm{GPA}\end{array}$ & $\begin{array}{c}C_{2}, \\
\mathrm{GPa}\end{array}$ & $\begin{array}{c}C_{3}, \\
\mathrm{~kJ} / \mathrm{g}\end{array}$ & $\begin{array}{c}E_{m}, \\
\mathrm{~kJ} / \mathrm{g}\end{array}$ \\
\hline$\alpha-\mathrm{Zr}$ & 37.859 & -111.1710 & 2296.4979 & -483.9864 & 0.0 \\
$\omega-\mathrm{Zr}$ & 38.746 & -94.2918 & 1944.8505 & -420.5750 & 0.01053 \\
$\beta-\mathrm{Zr}$ & 35.125 & -113.8496 & 2358.3400 & -458.8929 & 0.07519
\end{tabular}
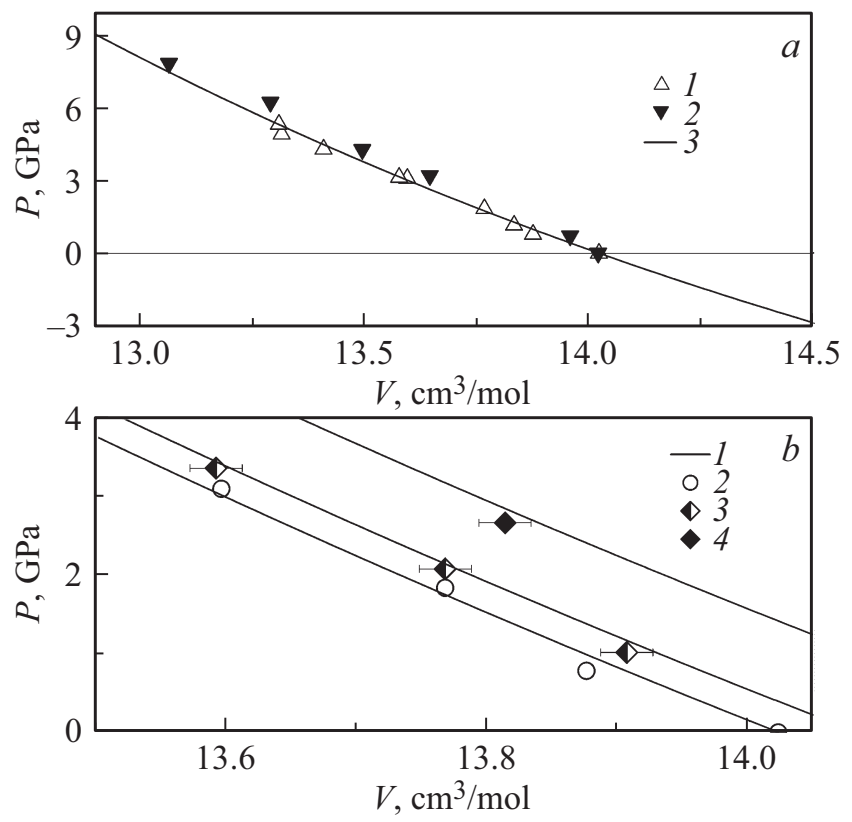

Рис. 6. Изотермы высокого давления (зависимость давления $P$ от объема $V$ ) при температуре $T_{i}=300 \mathrm{~K}$ полиморфной модификации циркония $\alpha-\mathrm{Zr}$. $a$ - комнатные изотермы: 1,2 - экспериментальные изотермы из [9] и [11] соответственно, 3 - расчетная изотерма с константами из пабл. 1,$2 ; b: 1-$ расчетные изотермы (снизу-вверх) при $T_{i}=300,473,918 \mathrm{~K}$, $2-4$ - эксперимент для $T_{i}=300,473,918 \mathrm{~K}$ из [9] соответственно.

Заметим далее, что построение полуэмпирических уравнений состояния циркония и соответствующие линии равновесия фаз циркония до высоких давлений и температур, где происходят $\alpha-\mathrm{Zr} \leftrightarrow \omega-\mathrm{Zr} \leftrightarrow \beta-\mathrm{Zr}$ полиморфные превращения этого металла предпринимались ранее в $[12,13]$. Однако представляется, что в настоящее время следует принимать во внимание результаты работ $[11,14]$, в которых обсуждается изоструктурный переход циркония $\beta-\mathrm{Zr} \leftrightarrow \beta^{\prime}-\mathrm{Zr}$ на комнатной изотерме при давлении $56-58 \mathrm{GPa}$. Действительно, согласно [14] линия равновесия $\beta-\mathrm{Zr} \leftrightarrow \beta^{\prime}-\mathrm{Zr}$ заканчивается критической точкой при давлении $\sim 50 \mathrm{GPa}$ и температуре $\sim 700 \mathrm{~K}$. Но это означает, что часть экспериментальных точек ударной адиабаты циркония принадлежат надкритической изоструктурной фазе $\beta^{\prime}$-Zr. Поэтому в данной работе уточнение величин $v_{x}$ для фаз высокого давления производился из условия наилучшего совпадения между их расчетными и экспериментальными ударными адиабатами, а не с изотермами высокого давления. Схема этого построения выглядит следующим образом.

Воспользуемся стандартной методикой физики ударных волн в виде решения системы уравнений (см., например, [15]), состоящей из калорического уравнения состояния (9) в обозначениях $E_{h}=E\left(V, T_{h}\right)$, где $T_{h}-$ температура на ударной адиабате, термического уравнения состояния (10) в обозначениях $P_{h}=P\left(V, T_{h}\right)$ и 
закона сохранения энергии на ударном скачке

$$
E_{h}-E_{0}=\frac{1}{2}\left(P_{h}+P_{0}\right)\left(V_{0}-V\right),
$$

где $E_{0}=E\left(V_{0}, T_{0}\right)$.

Система трех уравнений $(9),(10)$ и $(13)$, содержит три неизвестных $E_{h}, P_{h}$ и $T_{h}$, которые определяют термодинамическое состояние материала на ударной адиабате при заданном объеме $V$ и фиксированной величине $v_{x}$. Найденное решение этой системы наряду $E_{h}, T_{h}$ и $P_{h}$ позволяет рассчитать массовую скорость ударной волны как

$$
u=\sqrt{\left(P_{h}-P_{0}\right)\left(V_{0}-V\right)}
$$

и, тем самым, получить расчетную ударную адиабату материала не только в координатах давление $\left(P_{h}\right)-$ температура $\left(T_{h}\right)$, но в координатах массовая скорость $(u)$-давление $\left(P_{h}\right)$. Располагая расчетными ударными адиабатами материала при различных значениях величины $v_{x}$, мы можем выбрать то значение $v_{x}$, которому соответствует оптимальное взаиморасположение расчетной и экспериментальной ударной адиабаты в координатах скорость $(u)$-давление $(P)$ и, тем самым, определить параметр $v_{x}$. Эта процедура подгонки $v_{x}$ была выполнена для фаз высокого давления $\omega$-Zr- и $\beta$-Zr-фаз. Окончательные значения $v_{x}$ наряду с $V_{0}, v_{0}, \Theta_{0}, \beta_{0}$, а также $C_{1}, C_{2}, C_{3}$ и $E_{m}$ для $\omega$ - и $\beta$-фазы представлены в табл. 1 и 2 .

В заключение прокомментируем параметры фаз высокого давления циркония из табл. 1 и 2. Величины $V_{0}, v_{0}$, определялись с использованием результатов [9]. Вариацией величины $\Theta_{0}$ находилось нужное значение энтропии фаз. То есть величина $\Theta_{0}$ подбирались такой, чтобы скачок энтропии $\Delta S\left(T_{t r}, P_{t r}\right)$ в известных точках фазовых переходов $\beta-\mathrm{Zr} \leftrightarrow \beta-\mathrm{Zr}$ и $\alpha-\mathrm{Zr} \leftrightarrow \omega-\mathrm{Zr}$ был максимально близок к экспериментально измеряемой величине $\Delta S=\Delta V /(d T / d P)$, где согласно уравнению Клаузиуса-Клайперона $\Delta V$ - изменение молярного объема, $d T / d P$ - наклон линии равновесия. Данные для точек фазовых переходов $\alpha-\mathrm{Zr} \leftrightarrow \omega-\mathrm{Zr}$ и $\alpha-\mathrm{Zr} \leftrightarrow \beta-\mathrm{Zr}$ соответственно $T_{t r}=600 \mathrm{~K}, P_{t r}=4 \mathrm{GPa}$ и $T_{t r}=1136 \mathrm{~K}$, $P_{t r}=0 \mathrm{GPa}$ взяты из [16]. Параметры $\beta_{0}$ и $E_{m}$ для $\omega$-фазы и $\beta$-фазы рассматривались как подгоночные коэффициенты и подбирались так, чтобы химический потенциал

$$
\mu=\Phi(V, T)+P V
$$

в этих точках $\alpha$-фазы совпадал с химическими потенциалами $\omega$ - и $\beta$-фазы.

Верификация термодинамического описания циркония частично показана на рис. 5, на котором видно, что в координатах давление $(P)$-массовая скорость $(u)$ расчетная ударная адиабата циркония $P_{h}(u)$ для $\alpha-\mathrm{Zr}$ и $\beta-\mathrm{Zr}$ не выходит за пределы погрешностей экспериментальной ударной адиабаты этого металла из [7]. Кроме этого на рис. 6 представлены расчетные изотермы высокого давления $\alpha-\mathrm{Zr}$ и $\beta-\mathrm{Zr}$ при повышенных температурах вместе с экспериментальными данными $[9,11]$. Видно, что в области комнатных температур и при температуpe $918 \mathrm{~K}$ расчет для $\beta-\mathrm{Zr}$ совпадает с экспериментом в пределах разброса экспериментальных данных.

\section{2. Фазовая диаграмма циркония}

Как хорошо известно, знание свободной энергии фаз как функции своих переменных позволяет рассчитать линии равновесия между этими фазами. Техника расчета фазовой диаграммы с используемым в данной работе функциональным видом (1) свободной энергии заключается в следующем. Химический потенциал $\mu$ однокомпонентной системы вычисляется согласно формуле (15). При этом уравнение состояния (10) для каждой фазы позволяет исключить из (15) объем и представить химические потенциалы, например, $\alpha$ - и $\beta$-фазы в виде функций $\mu_{\alpha}(P, T)$ и $\mu_{\beta}(P, T)$ своих переменных давления $P$ и температуры $T$. Поэтому линия равновесия $T_{\alpha \beta}=T_{\alpha \beta}(P)$ между $\alpha$ - и $\beta$-фазой может быть рассчитана как линия пересечения поверхностей химических потенциалов этих фаз в пространстве $\{\mu, P, T\}$, задаваемая уравнением $\mu_{\alpha}(P, T)=\mu_{\beta}(P, T)$.

Определенные вышеописанным способом соответствующие линии равновесия фаз циркония показаны линиями 1 на рис. 7. На рис. 7 показаны также экспериментальные точки 2 линии равновесия $\omega-\mathrm{Zr} \leftrightarrow \beta-\mathrm{Zr}$ из [16].

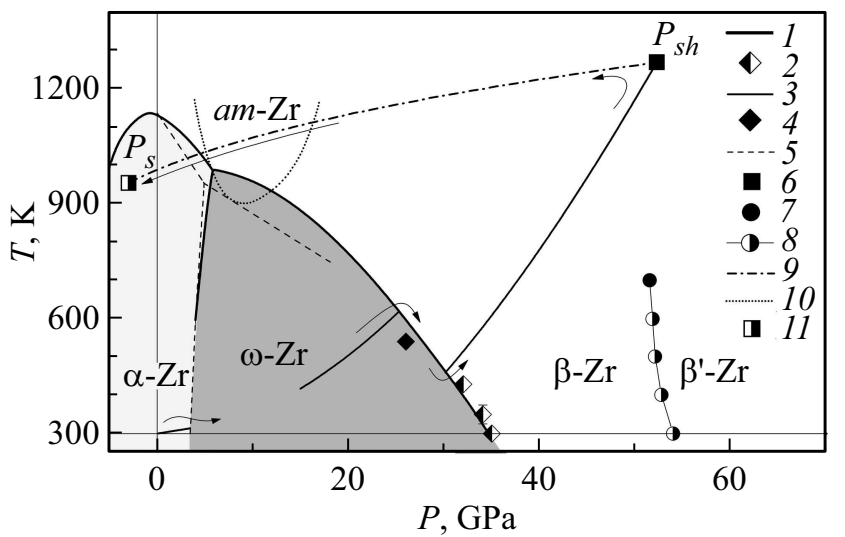

Рис. 7. Фазовая диаграмма циркония в координатах давление $R$-температура $T .1$ - линии равновесия $\alpha$-, $\omega$-, $\beta$-Zr-фаз, рассчитанные в данной работе, 2 - экспериментальные точки температуры и давления фазового перехода $\omega-\mathrm{Zr} \leftrightarrow \beta-\mathrm{Zr}$ из [16], 3 - расчетная ударная адиабата циркония, пересекающая линии равновесия фазовых превращений, 4 - давление и температура фазового превращения ударно-сжатого циркония из [7], 5 - линии равновесия циркония из [16], 6 - состояние $\left(P_{s h}, T_{s h}\right)$ ударно-сжатого циркония в экспериментах данной работы, 7 - критическая точка линии равновесия изоструктурного перехода [14], 8 - расчетная линия равновесия изоструктурного перехода $\beta-\mathrm{Zr} \leftrightarrow \beta^{\prime}-\mathrm{Zr}$ [14], $9-$ расчетная изэнтропа разгрузки $\beta-\mathrm{Zr}, 10-$ область аморфного циркония $a m-Z r$ из [16], 11 - состояние $\left(P_{s}, T_{s}\right)$ метастабильной фазы $\beta-\mathrm{Zr}$ в области растяжения (стрелками обозначен путь ударноволнового сжатия и последующей разгрузки). 
Как видно, расчетная линия равновесия $\omega-\mathrm{Zr} \leftrightarrow \beta-\mathrm{Zr}$ согласуется с экспериментом [16].

На рис. 7 представлена также расчетная ударная адиабата циркония 3 , пересекающая линии равновесия фаз в координатах давление температура $T_{h}\left(P_{h}\right)$. Как видно, 3 согласуется с оценками температуры и давления в точке излома ударной адиабаты циркония 4 из [8]. То есть расчеты ударных адиабат фаз циркония согласуются качественно и количественно с экспериментом, что свидетельствует в пользу достоверности разработанного термодинамического описания циркония при давлениях ударного сжатия до $\sim 100 \mathrm{GPa}$. В целом можно сказать, основные черты расчетной фазовой диаграммы циркония на рис. 7 согласуются с теоретическими и экспериментальными работами других авторов, например, 5 из [16], а также $[8,12,13]$.

Представленное полуэмпирическое описание позволяет обсудить термодинамическую историю ударного сжатия и последующей разгрузки циркония в выполненных экспериментах. Так, значение температуры ударного сжатия циркония $T_{s h}$ при давлении $P_{s h}=52 \mathrm{GPa}$ (см. 6 на рис. 7) оказывается равным $T_{s h}=1268 \mathrm{~K}$. Как видно точка $\left(T_{s h}, P_{s h}\right)$ расположена существенно выше критической точки 7 линии равновесия 8 изоструктурного перехода из [14].

Обсудим значение температуры циркония в разгрузке, когда развивается откольное разрушение в экспериментах по схеме рис. 3. На рис. 7 графиком 9 представлена расчетная изэнтропа разгрузки ударно-сжатой фазы $\beta-\mathrm{Zr}$, выходящая из точки $\left(T_{s h}, P_{s h}\right)$. Как видно, траектория 9 проходит через область аморфной фазы циркония 10 из [16] и затем, минуя область существования $\omega-\mathrm{Zr}$, пересекает линию равновесия $\alpha-\mathrm{Zr} \leftrightarrow \beta-\mathrm{Zr}$ при давлении $4.5 \mathrm{GPa}$ и температуре $1030 \mathrm{~K}$. В первом приближении, считая, что при пересечении области существования аморфной фазы и фазы $\alpha-\mathrm{Zr}$, цирконий остается в $\beta$-фазе даже в области растяжения, температура $T_{s h}$ циркония при значении растягивающего давления равного $P_{s}=-2.8 \mathrm{GPa}$ в точке 11 составляет $T_{s}=957 \mathrm{~K}$.

Однако если $\beta-\mathrm{Zr}$ фаза превращается в разгрузке в исходную $\alpha$-Zr-фазу, то температура разгруженного металла будет больше $T_{s}$, поскольку этот процесс идет с выделением тепла. Увеличение температуры в этом случае можно оценить, воспользовавшись соотношением $\Delta T=\Delta H / C_{p}$, где величина $\Delta H=3.94 \mathrm{~kJ} / \mathrm{mol}$ справочное значение энтальпии перехода $\alpha-\mathrm{Zr} \leftrightarrow \beta-\mathrm{Zr}$, а теплоемкость $C_{p}$ рассчитана согласно общей формуле

$$
C_{p}=-T\left(\frac{\partial^{2} \Phi}{\partial T^{2}}+\frac{(\partial P / \partial T)^{2}}{(\partial P / \partial V)}\right) .
$$

в точке $\left(T_{s}, P_{s}\right)$ и составляет $C_{p}=28.07 \mathrm{~J} / \mathrm{mK}$. Эти значения дают $\Delta T=140 \mathrm{~K}$. Таким образом, объединяя оценки $\Delta T$ и $T_{s}$, можно сказать, что в проведенных экспериментах температура циркония при его откольном разрушении составляла $T_{s} \approx 1027(70) \mathrm{K}$.

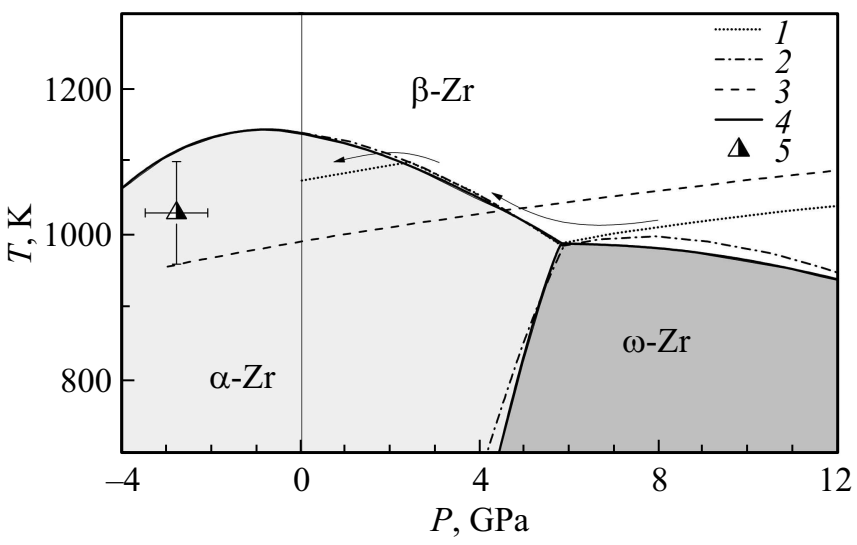

Рис. 8. Оценка температуры ударно-разогретого циркония при откольном разрушении. 1 и 2 - изэнтропа разгрузки (стрелками обозначен путь ударно-сжатого материала при разгрузке) и фазовая диаграмма циркония соответственно из [13], 3 и 4 - изэнтропа разгрузки и фазовая диаграмма данной работы, 5 - оценка параметров состояния циркония при откольном разрушении в проведенных экспериментах.

Отметим, что детальный количественный расчет разогрева ударносжатого циркония в разгрузке выполнен в [13]. В этой работе рассчитаны фазовая диаграмма циркония, его ударная адиабата и семейство изэнтроп разгрузки, пресекающих линии равновесия полиморфных модификаций. Часть результатов [13] представлена на рис. 8. Как видно, разогрев циркония вдоль изэнтропы 2, исходящей от давления ударного сжатия $49 \mathrm{GPa}$ в [13] составляет величину $150 \mathrm{~K}$, что оправдывает оценку $\Delta T=140 \mathrm{~K}$.

Полученная оценка температуры $T_{s}$ косвенно согласуется также с результатами исследования сохраненных образцов (см. рис. $1, b, 2, b$ и $c$ ). Действительно, вопервых, рентгенограмма 1 поверхности откола циркония на рис. $1, b$, совпадающая со справочной рентгенограммой 2 для $\alpha-\mathrm{Zr}$, свидетельствует о полном разрушении исходной [002] текстуры образца. Во-вторых, металлографический анализ откольной пластины на рис. 2, $c$ выявляет трехкратное измельчение зерна и формирование квазиизотропной мелкозернистой структуры. Но именно при температуре $\approx 1100 \mathrm{~K}$ наблюдаются аналогичные эффекты в известном способе термической обработки циркониевых сплавов (см., например, [17]), включающем быстрый нагрев этих сплавов до температур $1153-1173 \mathrm{~K}$ существования $\beta$-Zr-фазы, выдержку при этих температурах в течение $10-15 \mathrm{~s}$ и последующую закалку.

В заключение обсудим наличие максимума на линии равновесия $\alpha-\mathrm{Zr} \leftrightarrow \beta-\mathrm{Zr}$ в области отрицательных давлений. Эта особенность получена в результате экстраполяции в область растяжения полуэмпирических уравнений состояния, полученных на основании экспериментальных данных при сжатии. Поэтому существование максимума линии равновесия $\alpha-\mathrm{Zr} \leftrightarrow \beta-\mathrm{Zr}$ на рис. 8 
при отрицательном давлении $-0.8 \mathrm{GPa}$ и температуpe $1140 \mathrm{~K}$ носит гипотетический характер и нуждается в экспериментальной проверке.

\section{3. Математическое моделирование ударно-волновых экспериментов}

Полученные экспериментальные результаты и разработанные уравнения состояния могут быть применены к прикладной задаче описания и прогнозирования откольного разрушения циркония с помощью математического моделирования откола в условиях выполненных экспериментов. Моделирование осуществлялось в рамках одномерного гидрокода [18]. Требуемые в гидрокоде уравнения состояния материалов экспериментальной сборки рис. 3 (нержавеющей стали, плексигласа, тефлона, алюминия) взяты из [5]. Для циркониевого образца использовалось уравнение состояния $\beta$-Zr-фазы из табл. 1 и 2. Моделирование плексигласа и тефлона производилось в гидродинамическом приближении. Для металлических материалов использовалась упруго-пластическая модель в форме [19]. Параметры упруго-пластической модели для алюминия и нержавеющей стали взяты из [8], для циркония использованы данные из [1].

Откольное разрушение циркония моделировалось в рамках модели мгновенного откола. Считалось, что в лагранжевой частице образца мгновенно образуются две свободные поверхности, как только давление в этой частице достигнет значения $\sigma_{S}=-3.0 \mathrm{GPa}$. Было проведено также моделирование „безоткольных“ экспериментов, соответствующих бесконечно большой (по абсолютной величине) откольной прочности. Результаты моделирования представлены на рис. 4 .

Как видно, в случае моделирования экспериментов с простой преградой в постановке рис. 4, $a$, модельный профиль 3 на рис. 4, $a$ удовлетворительно воспроизводит откольный импульс в момент $t_{S}$ и последующую его циркуляцию в откольной пластине в виде ступенчатой разгрузки на экспериментальном профиле 1. Видно также существенное различие „безоткольного“ профиля 2 и экспериментального профиля 1 после момента $t_{S}$.

Модельный профиль 3 на рис. $4, c$, соответствующий усложненной преграде по схеме рис. $3, b$, также близок к экспериментальному профилю 1 . Отметим, что в этом случае модельные профили - „безоткольный“ 2 и профиль 3 , содержащий откольный импульс, незначительно различаются между собой. Однако качественное различие модельных профилей 2 и 3 позволяет идентифицировать малозаметную особенность на экспериментальном профиле 1 в области $t_{S}$ как откольное разрушение образца в усложненной постановке рис. $3, b$. Таким образом, рассматриваемая модель, во-первых, обладает прогностическими возможностями и, во-вторых, позволяет квалифицировать экспериментальные профили в постановке эксперимента рис. $3, b$ как экспериментальные данные по откольному разрушению образца циркония в усложненных условиях слоистой преграды.

\section{5. Заключение}

Проведены эксперименты по измерению откольной прочности образцов циркония марки 702 в плоской одномерной постановке. Амплитуда нагружающей ударной волны в цирконии составила величину 52(1) GPa. Расчетная температура ударного сжатия образцов составила $1268 \mathrm{~K}$. Откольная прочность циркония, предварительно сжатого и разогретого в ударной волне, составила величину $\sigma_{S}=-2.8(7) \mathrm{GPa}$ при температуpe $1027(70)$ К. Построены полуэмпирические уравнения состояния полиморфных модификаций циркония $\alpha-\mathrm{Zr}$, $\omega-\mathrm{Zr}$ и $\beta-\mathrm{Zr}$ и линии равновесия этих модификаций в области давлений до $\sim 50 \mathrm{GPa}$ и температур до $\sim 1200 \mathrm{~K}$. С использованием полученных экспериментальных и расчетных результатов проведено прогностическое моделирование откола в выполненных экспериментах.

\section{Финансирование работы}

Работа выполнена при поддержке Госкорпорации „Ро-

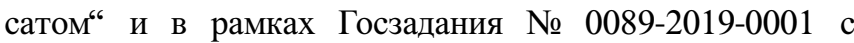
использованием оборудования уникальной научной установки „Экспериментальный взрывной стенд“.

\section{Конфликт интересов}

Авторы заявляют, что у них нет конфликта интересов.

\section{Список литературы}

[1] Д.Н. Казаков, О.Е. Козелков, А.С. Майорова, С.Н, Малюгина, С.С. Мокрушин, А.В. Павленко. Механика твердого тела 6, 68 (2014).

[2] P.J. Hazell, G.J. Appleby-Thomas, E. Wielewski, J.P. Escobedo. Phil. Trans. R. Soc. A 372, 20130204 (2014).

[3] А.М. Молодец, В.И. Лебедев, А.Н. Дремин. Физика горения и взрыва 25, 4, 101 (1989).

[4] D.E. Grady, M.E. Kipp. In: High-Pressure Shock Compression of Condensed Matter / Ed. J.R. Asay, M. Shahinpoor. Springer-Verlag, N.Y.-Berlin-Heidelberg (1992). P. 265.

[5] А.М. Молодец, А.А. Голышев. ФТТ 61, 8, 1492 (2019).

[6] А.А. Голышев, А.М. Молодец. ФГВ 49, 2, 106 (2013).

[7] R. McQueen, S. Marsh, J. Taylor, J. Fritz, W. Carter. In: High Velocity Impact Phenomena. High-Velocity Impact Phenomena / Ed. R. Kinslow. Academic Press, N.Y.-London (1970). P. 293.

[8] В.И. Романченко, Г.В. Степанов. ПМТФ 4, 141 (1980).

[9] Y. Zhao, J. Zhang, C. Pantea, J. Qian, L.L. Daemen, P.A. Rigg, R.S. Hixson, G.T. Gray III, Y. Yang, L. Wang, Y. Wang, T. Uchida. Phys. Rev. B 71, 184119 (2005).

[10] Л.В. Альтшулер, С.Б. Кормер, А.А. Баканова, Р.Ф. Трунин. ЖЭТФ 38, 3, 790 (1960).

[11] Y. Akahama, M. Kobayashi, H. Kawamura. J. Phys. Soc. Jpn 60, 10, 3211 (1991).

[12] S.A. Ostanin, V.Yu. Trubitsin. Phys. Rev. B 57, 13485 (1998).

[13] Е.А. Козлов, В.М. Елькин, И.В. Бычков. Физ. мет. и металловед. 82, 4, 22 (1996). 
[14] E. Stavrou, L.H. Yang, P. Söderlind, D. Aberg, H.B. Radousky, M.R. Armstrong, J.L. Belof, M. Kunz, E. Greenberg, V.B. Prakapenka, D.A. Young. Phys. Rev. B 98, 220101(R) (2018).

[15] А.М. Молодец, А.А. Голышев, Д.В. Шахрай. ЖЭТФ 151, 3, 550 (2017).

[16] J. Zhang, Y. Zhao, C. Pantea, J. Qian, L.L. Daemen, P.A. Rigg, R.S. Hixson, C.W. Greeff, G.T. Gray III, Y. Yang, L. Wang, Y. Wang, T. Uchida. J. Phys. Chem. Solids 66, 1213 (2005).

[17] В.Ф. Зеленский, А.И. Стукалов, И.М. Неклюдов, Г.Г. Гайдамаченко, В.М. Грицина, Н.М. Роенко, В.И. Савченко, Л.С. Ожигов, В.Н. Воеводин, Л.В. Платонов. Патент „Способ термической обработки циркониевых сплавов“ http://www.findpatent.ru/patent/176/1767924.html.

[18] В.В. Ким, А.А. Голышев, Д.В. Шахрай, А.М. Молодец. В сб.: Забабахинские научные чтения: сб. материалов XI Междунар. конф. (16-20 апреля 2012 г.) Изд-во РФЯЦ-ВНИИТФ, Снежинск (2012). С. 303.

[19] M.L. Wilkins. Computer simulation of dynamic phenomena. Springer, Berlin (1999). P. 83.

Редактор Д.В. Жуманов 